\title{
A Rare Case of Emphysematous Endocarditis Caused by Escherichia coli
}

"Ahmed S. Al-Dhahli, ${ }^{1}$ Rashid S. Al-Umairi, ${ }^{2}$ Osama Elkadi ${ }^{1}$

\begin{abstract}
Infective endocarditis (IE) is an infection of the heart endocardium with significant morbidity and mortality. Gram negative infection, particularly emphysematous IE, is an extremely rare and life-threatening disease. We report a 59-year-old diabetic female patient who was admitted to a secondary care hospital in Rustaq, Oman, in 2017 with the diagnosis of pneumonia for which she was started on antibiotics. Shortly afterwards, she developed facial and mouth deviation and became more tachypneic. Computed tomography of the brain demonstrated bilateral multiple small infarcts. Pulmonary angiography computed tomography was performed which ruled out pulmonary embolism. Nonetheless, it revealed an air-containing lesion around the mitral valve. Transthoracic echocardiography demonstrated a hyperechoic mobile lesion related to the mitral valve. Blood culture grew Escherichia coli and the diagnosis of E. coli emphysematous IE was made based on modified Duke criteria. The patient's clinical condition deteriorated and she suffered cardiac arrest leading to her death. The recommended treatment for non-HACEK IE includes an extended antibiotic course and surgery for selected patients.
\end{abstract}

Keywords: Endocarditis; Escherichia coli; Mitral Valve; Echocardiography; Intracranial Embolism; Emphysema; Case Report; Oman.

$\mathrm{I}$ NFECTIVE ENDOCARDITIS (IE) IS A LIFEthreatening condition which is defined as an infection of the cardiac endocardium. The valves are the most common cardiac structure affected by the disease. IE is the most prevalent infection of the cardiovascular system. ${ }^{1}$ Patients can present with constitutional symptoms including fever, chills and weight loss. Other clinical presentations of the disease are related to cardiac involvement including heart failure, vasculitis and systemic embolic phenomena. ${ }^{2}$

IE is caused by gram positive organisms in the majority of patients; staphylococci, streptococci and enterococci are responsible for more than $80 \%$ of IE cases. ${ }^{3}$ Rarely, IE can be caused by a group of gram-negative organisms other than Haemophilus species, Actinobacillus actinomycetemcomitans, Cardiobacterium hominis, Eikenella corrodens or Kingella kingae (HACEK). ${ }^{4,5}$ In older publications, IE caused by non-HACEK gram-negative organisms was predominantly seen among intravenous (IV) drug users. ${ }^{6}$ However, more recent studies have reported that the majority of non-HACEK IE was among patients with implanted endovascular devices, including prosthetic valves, permanent pacemakers and implantable cardioverter-defibrillators. ${ }^{6}$

Likewise, E. coli IE is rare with limited number of reported cases in the literature and it is usually associated with a higher mortality rate compared to HACEK gram-negative IE. ${ }^{4-6}$ The reported inpatient mortality rate is $24 \%$ for non-HACEK IE compared to 4\% for HACEK IE. ${ }^{7,8} \mathrm{Al}$ Abri et al. has reported 4.1\% incidence of E.coli IE in Oman. ${ }^{9}$ Emphysematous type of IE is extremely rare with very limited reported cases in the literature. ${ }^{3,10-12}$

\section{Case Report}

A 59-year-old female patient presented to the Emergency Department at a secondary care hospital in Rustaq, Oman, in 2017 with a five-day history of cough, shortness of breath, fever and chest discomfort. The patient had a medical history of uncontrolled diabetes, hypertension and ischaemic heart disease. Her echocardiography 10 years prior to admission demonstrated left ventricular hypertrophy with a normal left ventricle ejection fraction. There was no mitral or tricuspid valve regurgitation.

On physical examination, she was conscious, alert and oriented. Her vital signs were as follows: blood pressure of $102 / 60 \mathrm{mmHg}$, body temperature of $38^{\circ} \mathrm{C}$, heart rate of 109 beat per minute, $\mathrm{O}_{2}$ saturation of $90 \%$ and a random blood sugar of $19.3 \mathrm{mmol} / \mathrm{L}$. Chest auscultation revealed bilateral fine crepitation which was more prominent on the right. The remainder of the systemic examination was unremarkable.

Laboratory investigations showed high white blood cell counts at $17.8 \times 10^{3} / \mathrm{uL}$ (normal range: $2.4-9.5 \times 10^{3} / \mathrm{uL}$ ) with $81 \%$ neutrophil predominance, high glycosylated haemoglobin at 9.6\% (normal range: 4.8-6\%) and high troponin $\mathrm{T}$ at $153.1 \mathrm{pg} / \mathrm{mL}$ (normal range: $0-14 \mathrm{pg} / \mathrm{mL}$ ). Platelet count, haemoglobin, 


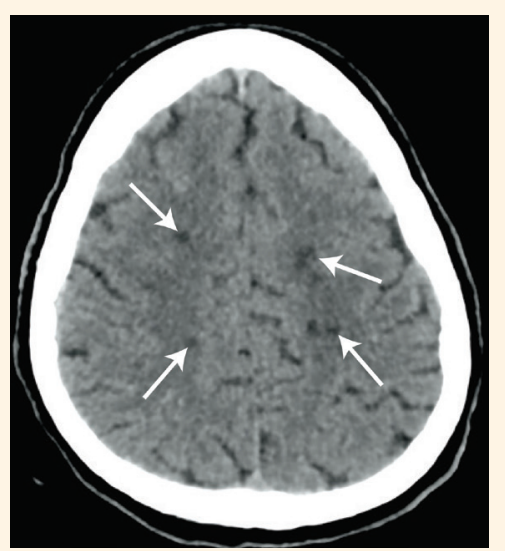

Figure 1: Non-contrast enhanced computed tomography of the brain of a 59-year-old female patient showing bilateral ill-defined small hypodense foci in the bilateral centrum semiovale and corpus callosum (not shown) suggestive of acute ischaemic infarction (arrows).

creatinine and urea levels were normal. Her chest radiograph showed a patchy opacity which was interpreted as pneumonic consolidation for which the patient was started on ceftriaxone.

On the second day of admission, the patient's Glasgow Coma Scale score deteriorated as she was responsive only to pain stimuli. Subsequently, she developed facial and mouth deviation, so a noncontrast enhanced computed tomography (CT) of the brain was performed using a 64-slice CT machine (Philips, Amsterdam, The Netherlands) that demonstrated multiple small subtle hypodense foci in both the centrum semiovali and corpus callosum [Figure 1]. The distribution and characteristics of the hypodensities were suggestive of acute ischaemic infarctions due to thromboembolic causes.

On the third day of admission, the patient was persistently febrile despite antibiotic administration with a blood pressure drop to $87 / 50 \mathrm{mmHg}$. Pulmonary embolism (PE) was suspected based on high D-dimer and worsening tachypnoea. Subsequently, a pulmonary angiography computed tomography (CTPA) was performed on a 64-slice CT machine (Philips). The procedure was performed craniocaudally with the following parameters: $0.625 \mathrm{~mm}$ detectors, auto$\mathrm{mA}$ (average of $224 \mathrm{~mA}$ ), $120 \mathrm{kVp}$ and a $512 \times 512$ matrix. The study was done with IV contrast material utilising PE protocol. Coronal and sagittal reformats were reconstructed. The study ruled out PE, but it demonstrated an air-containing lesion around the mitral valve [Figure 2]. There was mitral valve annulus calcification. Transthoracic echocardiogram was performed which revealed mild mitral regurgitation with a mobile hyperechoic mass related to the anterior mitral valve leaflet associated with a posterior acoustic shadow [Figure 3]. The left ventricle ejection fraction was $25 \%$. Blood culture from a single blood sample yielded growth of gram negative bacilli Escherichia coli and consequently the antibiotic was changed, based on the sensitivity profile, to piperacillin/tazobactam (4.5 g three times/day) on the third day of admission. A diagnosis of IE was established based on modified Duke clinical criteria. ${ }^{8}$

Nevertheless, the patient's clinical condition deteriorated on the fourth day of admission and eventually she suffered a cardiac arrest with ventricular arrhythmia then went into asystole leading to her death after a short period of restoration of normal sinus rhythm.

\section{Discussion}

In this case report, we described a patient with uncontrolled diabetes who was admitted with an impression of pneumonia and who was later on found to have emphysematous IE which ultimately led to the patient's death. Although, it was not possible to obtain a tissue sample to confirm the diagnosis of IE, the diagnosis of possible IE was established based on the modified Duke criteria which includes one major criterion (endocardial involvement as evident in echocardiogram as a lesion attached to the mitral leaflet) and two minor criteria (persistent fever and vascular phenomenon of multiple small

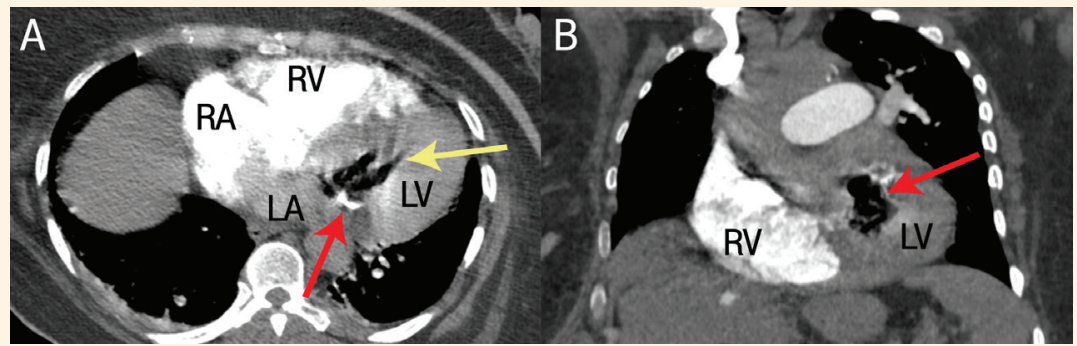

Figure 2: A: Axial pulmonary angiography computed tomography showing an air-containing lesion around the mitral valve (yellow arrow); note is made of mitral annual calcification (red arrow). B: Coronal reformat demonstrating the air density lesion in the mitral valve (red arrow).

$R A=$ right atrium; $R V=$ right ventricle; $L V=$ left ventricle; $L A=$ left atrium. 


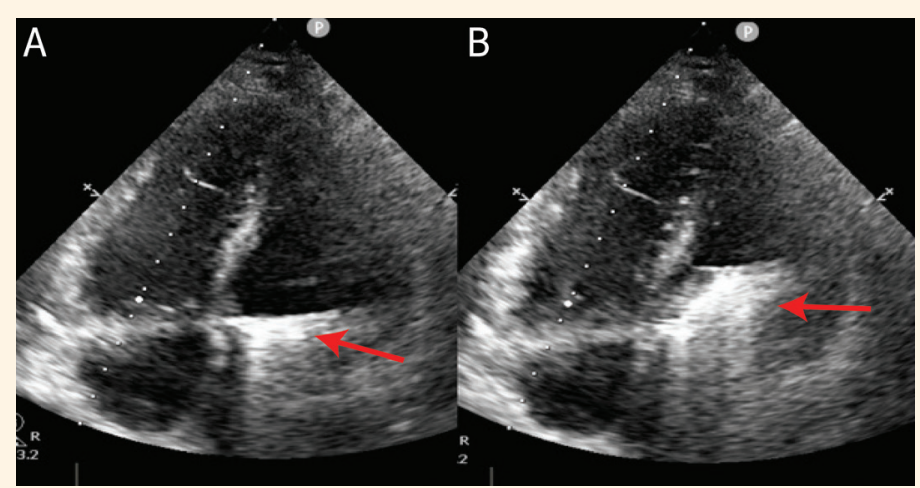

Figure 3: A: Four chamber view echocardiogram in the systole showing a hyperechoic lesion related to the anterior leaflet of the mitral valve (arrow). B: Redemonstration of the mobile hyperechoic lesion related to the mitral valve in the diastole (arrow).

embolic infarcts in the brain). ${ }^{8}$ In the current case, the positive blood culture (from a single blood sample) revealed the growth of $E$. coli which is an atypical microorganism for IE. No significant bacterial growth could be obtained from urine culture and no other source of infection could be identified.

E. coli is the most common non-HACEK gramnegative organism that cause IE, accounting for approximately one third of the cases. ${ }^{10}$ IE secondary to E. coli is usually associated with higher mortality and morbidity compared to IE due to HACEK gramnegative organisms. The source of IE secondary to E. coli is usually urinary tract infection. ${ }^{4,5}$ Diabetes, malignancy, excessive alcohol consumption and haemodialysis are considered to be risk factors for developing IE secondary to E. coli. ${ }^{6}$

In the present case, an air-containing lesion was seen around the mitral valve on CTPA indicating emphysematous type of IE (CT is the radiological gold standard tool for the diagnosis of emphysema). Literature review revealed two reported cases of $E$. coli related emphysematous IE. ${ }^{3,12}$ There are two additional emphysematous IE cases reported in the literature caused by Finegoldia magna and Citrobacter koseri. ${ }^{10,11}$ Previous case reports share similar radiological findings with the present case of air density vegetation around the mitral valve. Furthermore, all patients in these case reports had diabetes mellitus including the present patient., ${ }^{3,10-12}$

Choosing the appropriate antibiotic treatment for non-HACEK gram-negative-related IE is based on sensitivity profile of the responsible pathogen. ${ }^{13}$ Treatment by a combination of antibiotics with $\beta$-lactams (penicillins, cephalosporins or carbapenems) and either an aminoglycoside or fluoroquinolone for six weeks is considered appropritate. ${ }^{8}$ An infectious disease expert opinion in IE should be obtained due to known antibiotic resistance in non-HACEK organisms. ${ }^{8}$ Some patients in this group may require surgical intervention especially for Pseudomonasrelated left-sided IE. ${ }^{8,13}$

\section{Conclusion}

Emphysematous infective endocarditis caused by $E$. coli is an extremely rare disease that is associated with increased mortality and morbidity. Therefore, clinicians should be aware of this entity and pay close attention to the clinical course of patients with E. coli IE during hospitalisation in order to reduce mortality and morbidity related to the disease.

\section{AUTHORS' CONTRIBUTION}

ASA-D provided the diagnosis of the patient. ASA-D, RA-U and OE drafted the manuscript. RA-U conducted the literature review. OE provided the echocardiography images. All authors approved the final version of the manuscript.

\section{References}

1. Yusuf SW, Sharma J, Durand JB, Banchs J. Endocarditis and myocarditis: A brief review. Expert Rev Cardiovasc Ther 2012; 10:1153-64. https://doi.org/10.1586/erc.12.107.

2. Ferro JM, Fonseca AC. Infective endocarditis. Handb Clin Neurol 2014; 119:75-91. https://doi.org/10.1016/B978-0-70204086-3.00007-2.

3. Kim CJ, Yi JE, Kim Y, Choi HJ. Emphysematous endocarditis caused by AmpC beta-lactamase-producing Escherichia coli: A case report. Medicine (Baltimore) 2018; 97:e9620. https://doi. org/10.1097/MD.0000000000009620.

4. Chambers ST, Murdoch D, Morris A, Holland D, Pappas P, Almela M, et al. HACEK infective endocarditis: Characteristics and outcomes from a large, multi-national cohort. PLoS One 2013; 8:e63181. https://doi.org/10.1371/journal.pone.0063181.

5. Morpeth S, Murdoch D, Cabell CH, Karchmer AW, Pappas P, Levine D, et al. Non-HACEK gram-negative bacillus endocarditis. Ann Intern Med 2007; 147:829-35 https://doi.org/10.7326/0 003-4819-147-12-200712180-00002. 
6. Akuzawa N, Kurabayashi M. Native valve endocarditis due to Escherichia coli infection: A case report and review of the literature. BMC Cardiovasc Disord 2018; 18:195. https://doi. org/10.1186/s12872-018-0929-7.

7. Chambers ST, Murdoch D, Morris A, Holland D, Pappas P, Almela M, et al. HACEK infective endocarditis: Characteristics and outcomes from a large, multi-national cohort. PLoS One 2013; 8:e63181. https://doi.org/10.1371/journal.pone.0063181.

8. Baddour LM, Wilson WR, Bayer AS, Fowler VG Jr, Tleyjeh IM, Rybak MJ, et al. Infective endocarditis in adults: Diagnosis, antimicrobial therapy, and management of complications: A scientific statement for healthcare professionals from the american heart association. Circulation 2015; 132:1435-86. https://doi. org/10.1161/cir.0000000000000296.

9. Al Abri SS, Zahedi FI, Kurup PJ, Al-Jardani AK, Beeching NJ. The epidemiology and outcomes of infective endocarditis in a tertiary care hospital in Oman. J Infect Public Health 2014; 7:400-6. https://doi.org/10.1016/j.jiph.2014.04.004.
10. Cohen M, Stephens EH, Garshick M, Wang SH, Frager SZ, Mautner L, et al. Native valve emphysematous endocarditis caused by finegoldia magna in a novel pathogenic role. Infect Dis Clin Practice 2016; 24:57-9. https://doi.org/10.1097/IPC.0000000000000294.

11. Kesler S, Kim H, Perlman D, Dincer HE, Thenappan T, Tomic R. Air in the left ventricle. An unusual case of endocarditis. Am J Respir Crit Care Med 2016; 193:1176-7. https://doi.org/10.1 164/rccm.201506-1156IM.

12. Law D, Thomas M. Escherichia coli emphysematous endocarditis. Lancet Infect Dis 2020; 20:381. https://doi.org/10.1016/S14733099(19)30700-5.

13. Sexton DJ, Chu VH. Antimicrobial therapy of left-sided native valve endocarditis. From https://www.uptodate.com/contents/ antimicrobial-therapy-of-left-sided-native-valve-endocarditis Accessed: Jan 2021 